\title{
Tooth autotransplantation: a neglected approach?
}

\author{
A. Morel*1, J. Hamon², A. Lim³, D. Brézulier ${ }^{4}$, V. Fau', O. Sorel ${ }^{5}$ \\ 1 Diploma of University Specialist Degree in Orthodontics and Dentofacial Orthopedics, University \\ of Rennes 1, Rennes University Hospital \\ 2 Diploma of Specialized Studies of Oral Surgery University of Rennes 1, Rennes University \\ Hospital \\ ${ }^{3}$ Diploma of Specialized Studies of Oral Medicine, University of Rennes 1, Rennes University \\ Hospital \\ 4 University Hospital Assistant, University of Rennes 1, Rennes University Hospital \\ ${ }_{5}$ University Professor-Hospital practitioner, University of Rennes 1, Rennes University Hospital
}

\section{ABSTRACT}

Autotransplantation is a still unknown therapeutic solution that presents multiple indications in the growing patient. It requires close collaboration between the orthodontist, the dentist, and the oral surgeon. We will illustrate this article by a clinical case of transplantation of a premolar in place of a central incisor after a review of the indications, advantages, and surgical procedure of this method.

\section{KEYWORDS}

Dental transplantation, traumatism, orthodontics

\section{INTRODUCTION}

The earliest reports of tooth transplantation date back to ancient Egypt where slaves were forced to give their teeth to their pharaohs. However, allotransplantation posed histocompatibility problems. Autotransplantation or autogenous transplantation is a surgical procedure that involves the movement of one tooth from one position to another within the same person. This technique was first described in 1954 by Mr. L. Hale. The reaction of the dental tissues after autotransplantation has been described in detail by Andreasen et al., who demonstrated the possibility of a resumption of radicular edification and revascularization in immature teeth.

Although there are multiple indications and numerous advantages to autotransplantation, it is still not widespread, and perhaps wrongly so.

\section{Address for correspondence:}




\section{THE ROLE OF AUTOTRANSPLANTATION}

\section{Indications}

There are numerous indications for autotransplantation, all of which have the ultimate purpose of replacing a tooth. The major indicators being tooth loss because of trauma or carious lesions. Since the 1970s, Slagsvold and Bjercke have advocated for the autotransplantation of immature premolars to replace an incisor that was either missing or lost during trauma. Maxillary incisors are the most susceptible to bumper impact trauma, making them the teeth most susceptible to injury, comprising approximately $80 \%$ orthodontic trauma cases $^{8}$. The teeth best suited to replace a central incisor are the first and second mandibular premolars and the second maxillary premolar because their radicular anatomy is the most similar to that of incisors ${ }^{10}$. In the context of lateral incisor agenesis, Antonarakis et al. propose autotransplantation as an alternative to fixed prosthetic treatments ${ }^{6}$. They claim that this solution has the most favorable cost/benefit ratio. This solution is illustrated by Intra et al., who point out, based on their experience in a 13-year-old patient, that one of the expected benefits is the maintenance of the natural growth of the alveolar process compared to the placement of an implant ${ }^{15}$.

The replacement of molars, whose most frequent cause of avulsion remains carious lesions, is most often achieved via the autotransplantation of the third molars ${ }^{23,24}$. However, is the ideal age for harvesting the third molar for autotransplantation remains debated. According to the literature, it is also possible to transplant third molar germs ${ }^{7,9}$ as well as mature third molars on the $\operatorname{arch}^{24}$. Yu et al. have concluded that the autotransplantation of third molars to completely edified roots is reliable, whether the recipient site is a fresh extraction alveolus or a surgically created alveolus ${ }^{25}$.

More generally, transplantations are also indicated in any of the following cases: endodontic lesions, coronal or radicular fractures, root resorptions, aggressive periodontitis, tumors ${ }^{19}$, or during the impaction or ectopic eruption of maxillary canines ${ }^{17}$.

In cases where transplantation is not immediately possible (e.g., in cases where preliminary orthodontic treatment is required), cryopreservation and storage of the tooth in a tooth bank offers new possibilities ${ }^{2}$.

In fact, in the 1960s and 1970s, various authors, such as Bartlett and Reade, showed that after cryopreservation and transplantation, $86 \%$ stem cells resumed their development and produced dental tissues. Schwartz, Andreasen, and Kaku have used this method to cryopreserve mature teeth ${ }^{16}$.

\section{Contraindications}

The major contraindications are a lack of proper oral hygiene and the impossibility of ensuring a regular follow-up. A relative contraindication is the inadequate width of the alveolar bone at the recipient site, considering that modern grafting techniques can overcome this problem. The type of graft to put in place is debatable. Zufia et al. went 
as far as suggesting the concomitant transplantation of a third molar and its vestibular cortical bone to replace a second molar ${ }^{26}$.

\section{Advantages}

There are numerous esthetic, functional, and physiological advantages for autotransplantation. Unlike prosthetic implants, this technique also allows the alveolar bone to continue its growth, especially vertical ${ }^{21}$. Studies have shown that this phenomenon occurs both in the anterior region as well as in the transplanted molars, as long as the integrity of the periodontal ligament is maintained ${ }^{18}$. This makes the technique a perfect solution for young patients who are still in the growth phase.

Additionally, various studies have reported a $75 \%$ satisfaction rate among patients regarding the esthetic result after reconstitution by composite or ceramic crown ${ }^{12}$.

According to the authors, the success rate varies between $74 \%$ and $100 \%$ and mostly depends on the stage of radicular edification. These studies have shown that a resumption of radicular edification and revascularization were possible ${ }^{2}$ after transplantation.

In their study, Czochrowska et al. reported $90 \%$ and $74 \%$ success rate at 17 and 41 years after treatment ${ }^{11}$, which makes this method at least as effective as traditional prosthetic or implant solutions. The cost is also a deciding factor for a number of patients. Transplantation represents a cost-effective alternative to prostheses.

The major disadvantage, however, is the limited number of available transplants.

\section{THE OPERATIVE TECHNIQUE OF AUTOTRANSPLANTATIONS}

The success of dental replacement therapy by autotransplantation is determined by the quality of the residual periodontal tissues in the root surface of the tooth to be transplanted. The quality of this tissue depends on the graft-harvesting conditions.

Thus, radicular anatomy is a key factor because it allows the practitioner to anticipate the phases of treatment as well as any difficulties associated with the procedure.

An important point to note is that the functional periodontum is predominantly made up of fibroblasts positioned perpendicularly to the dental walls, thus offering a higher resistance to dislocation forces. Andreasen 3,4 demonstrated that periodontal cellular bodies were more resistant to tensile and shear forces than those under compression $^{13}$. Indeed, the latter tend to cause the explosion of cellular bodies against the alveolar and radicular walls. Therefore, the surgeon will check for a tearing or shearing effect on the periodontium using rotating or tensile dislocation movements rather than a pendulum movement, which may crush the periodontium I. A single, conically-shaped root, allowing dislocation via rotational movements along its major axis, is the most favorable situation ${ }^{4}$. In contrast, the presence 
of multiple and divergent roots on the dental graft makes the procedure difficult to perform (difficult extraction) and also complicates the healing process.

This surgery is most often performed under local or locoregional anesthesia. Meticulous antisepsis must be ensured. The described intervention takes place in two phases. The initial phase consists of the avulsion of the pathological tooth, the modification of its alveolus, and the extraction followed by the fitting of the graft. The second operative phase, which consists of the actual transplantation, will be performed 15 days later.

\section{First operative phase}

Thus, the pathological tooth is carefully and nontraumatically avulsed; the aim being to preserve the vestibular and palatal bone tables as much as possible. The tooth's alveolus is then prepared. For a multi-rooted tooth, the next step is the removal of the inter-radicular septum using gouge forceps. Then, the dimensions of the alveolus are expanded using different implant drills according to the size and shape of the transplant. The use of calibrated drills of various sizes makes it possible to adapt the drilling depth to the height of the graft that will be placed during the second operative phase.

According to the protocol defined by Gault $^{14}$ the graft is avulsed during this operative phase. This will allow us to test it and adapt the shape of the alveolus to the anatomy of the tooth to be transplanted. Between each fitting, the graft is placed back in its original alveolus. When the graft can be placed new alveolus without friction, it will then be returned to its original site for a period of healing, which will induce a cicatricial inflammatory reaction in the periodontium to be transplanted.

\section{Second operative phase}

This will be performed approximately 15-21 days after the first operation. After local or locoregional anesthesia of the donor and recipient sites, a sulcal incision is performed with a cold blade is performed at the collar of the tooth to be removed along with those of neighboring teeth. A discharge can be achieved by sparing the interdental papillae and the zenith of the collars. This incision will improve visibility of the surgical site while ensuring flap vascularization. The mucoperiosteal detachment will be performed with a sickle syndesmotome or surgical scraper.

An incision is also made on the edentulous ridge of the recipient site and in the sulci of the neighboring teeth. A discharge can also be performed and the mucoperiosteal detachment that will allow complete vision of the new alveolus. The tooth is then avulsed using forceps or an elevator via rotational or pulling movements. Pendular movement should not be used to avoid crushing the periodontal fibers. This avulsion must cause little to no trauma to the periodontal ligament.

Note that avulsion may be complicated and may require an alveolectomy. The latter will be performed using a round bur under constant irrigation, and it will target the alveolar bone while ensuring safety of the tooth or the periodontal ligament.

We will then proceed to graft placement in its new alveolus. This must be done gently. The tooth must then 
be placed under slight occlusion. Any occlusal overload can compromise the success of the protocol. While repositioning the tooth in its alveolus, a biological space of approximately $2 \mathrm{~mm}$ must be maintained between the cementoenamel junction and the height of the alveolar ridge.

The flap of the recipient site is then positioned optimally to ensure proper collar alignment. Simple Vicryl ${ }^{\mathrm{TM}} 4.0$ sutures are used on the flap as well as the donor site.

Finally, to ensure compression, a cross suture restraint is performed on the tooth with nonabsorbable suture thread.

\section{Postoperative follow-up}

Postoperatively, a combination of analgesics, anti-inflammatories, and mouthwashes will be prescribed for 8 days. The patient will maintain a semisolid diet for several days. Proper oral hygiene is essential and should be rigorous using a $7 / 100^{\text {th }}$ postoperative toothbrush. Suture removal can be performed within 8 days and the

\section{CLINICAL CASE}

Romain, aged 13 years, came in for consultation in June 2016. Owing to trauma experienced in 2012, his left maxillary central incisor (tooth 21) was expelled and then re-implanted. The patient's complaints were mostly esthetic in nature.

An intraoral examination of the maxilla revealed ankylosis of tooth 21 . This resulted in impeded vertical growth, compression suture will be placed after 3 weeks. Several postoperative clinical and radiological tests may be scheduled at weeks 1 and 3 , then at months 3 and 6 , and ultimately every year.

It should be noted that during the transplantation of an immature tooth, the follicular sac must be preserved. It will then be transplanted with the tooth.

This sac will facilitate the finalization of radicular edification and the preservation of its periodontal integrity. It will also play an important role in the pulpar revascularization process of the transplant $^{20}$. In the case of a mature transplant, revascularization is much more complicated and often results in pulpar necrosis. Devitalizing the graft should then be considered ${ }^{1,5}$. This will be done before transplantation when the tooth is on the arch. Devitalization of the graft outside the alveolus should be avoided to preserve the periodontal ligament. In the case of impacted teeth, root canal treatment will be done 3 weeks after transplantation on a partially stabilized tooth.

which explains the unsightly appearance of the smile.

The patient presents with a bilateral Angle Class-I occlusion with a left Class-II tendency (Fig. 1a, 1b).

Among the additional examinations, a cone-beam computed tomography was performed, which revealed the absence of a vestibular cortex with respect to 21. Moreover, external root 
resorptions were visible. In light of all these data, the tooth was deemed nonpreservable (Fig. 2a, 2b, 2c).

Given the impossibility of implant placement because of the patient's age and his family's refusal, the proposed treatment plan involved the avulsion of a maxillary premolar, followed by the establishment of a unilateral therapeutic Class-II malocclusion. It was then decided to transplant 25 into the place of 21 with the closure of sector-2 space
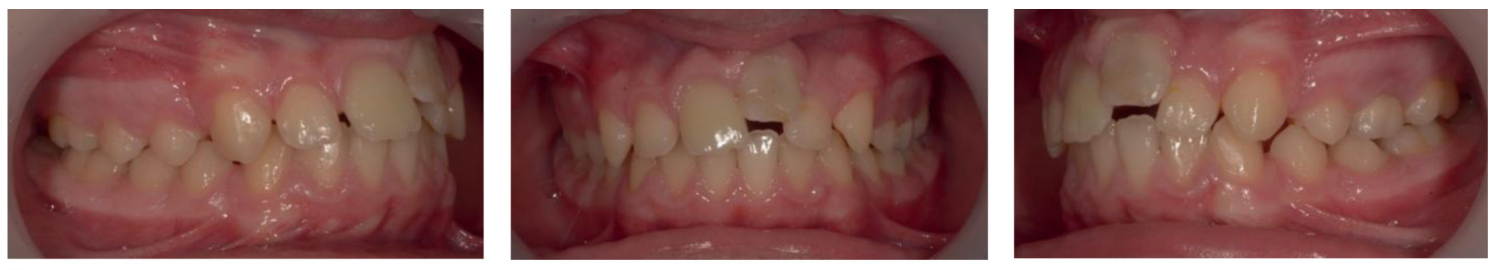

a

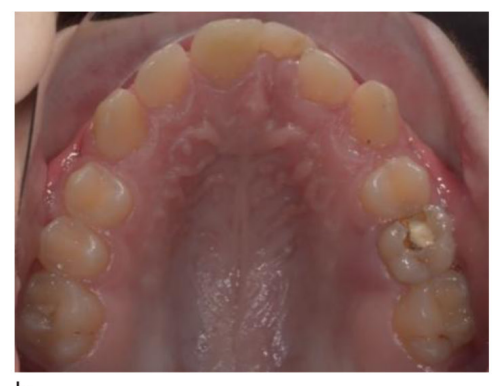

Figure 1

(a) Intraoral view of interarch relationships. (b) Intraoral view of maxillary arch.

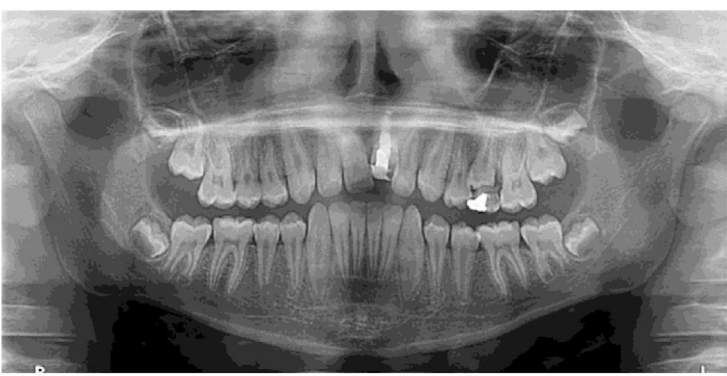

a

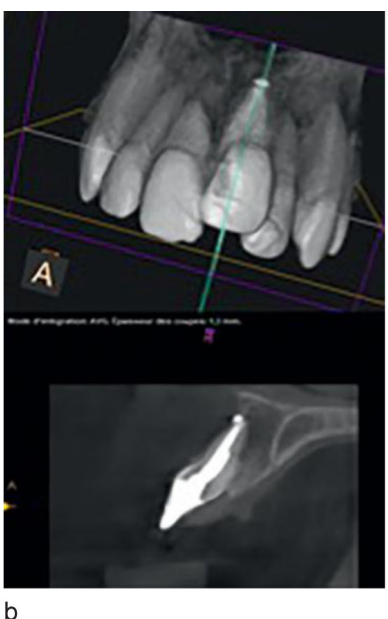

b

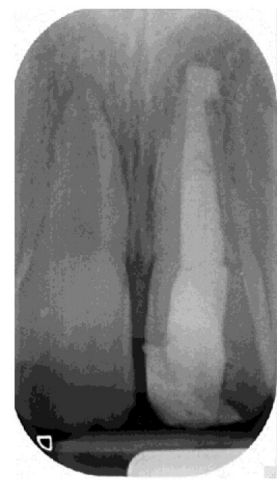

C

Figure 2

(a) Panoramic radiograph of start of treatment. (b) Three-dimensional reconstitution showing areas of root resorption. (c) Retroalveolar radiograph, note the areas of resorption surrounding the root 
and the establishment of a therapeutic Class-II malocclusion on the extraction side. A multibracket appliance treatment is set up to expand the anterior space of the recipient site. Tooth 25 was depulped before its transplantation, and the radicular edification was completed (Fig. 3a, 3b).

The transplantation protocol took place in two phases-21 was extracted and the premolar transplant was performed 15 days later in the alveolus of 21, which was previously adapted.
The tooth was positioned at a $90^{\circ}$ angle so that the mesiodistal diameter of the premolar resembled that of an incisor.

In such cases, soft-tissue management is crucial. A flap that was repositioned during the surgery ensures proper alignment of the collars. Particular care should be taken to preserve the periodontal ligament, as this is crucial for the success of the surgery (Fig. 4a, 2b).

A composite reconstruction is performed 2 months after the transplantation to restore the esthetic aspect.

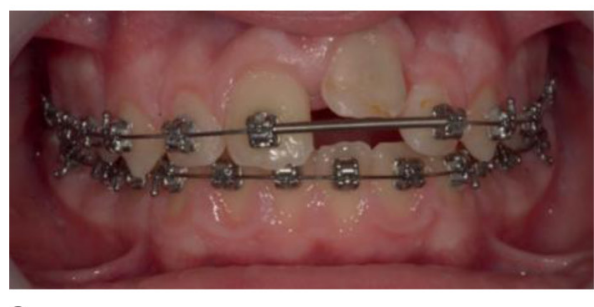

a

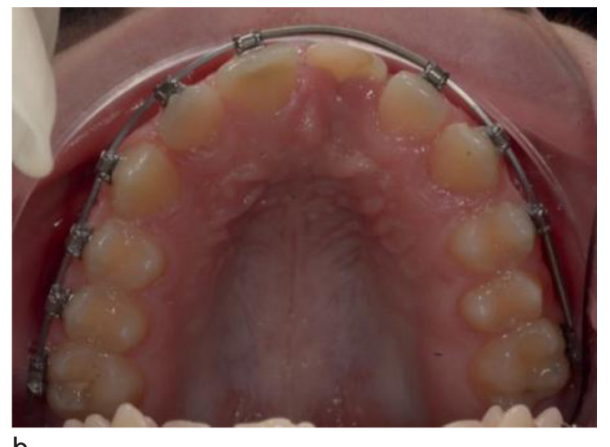

b

Figure 3

(a) End of orthodontic preparation, note the significant space gained at the implant site.

(b) Occlusal view after preparation for transplantation

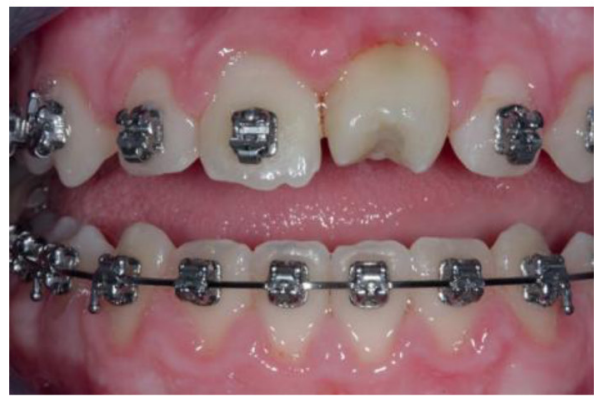

a

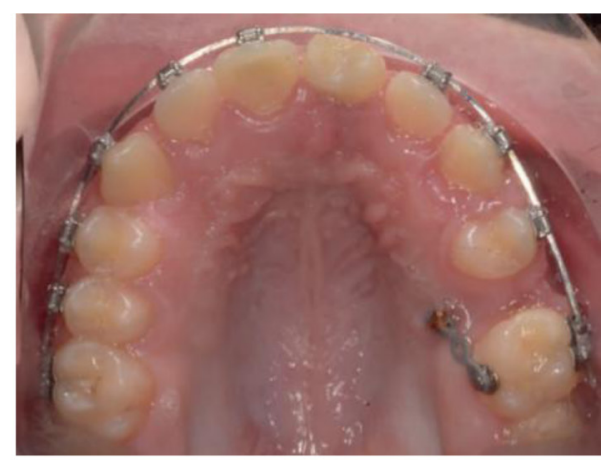

b

Figure 4

(a) Frontal view 1 month after transplantation. The tooth is positioned with a $90^{\circ}$ rotation ensuring that the emerging profile is compatible with the coronal reconstitution of a central incisor. (b) Occlusal view after transplantation 

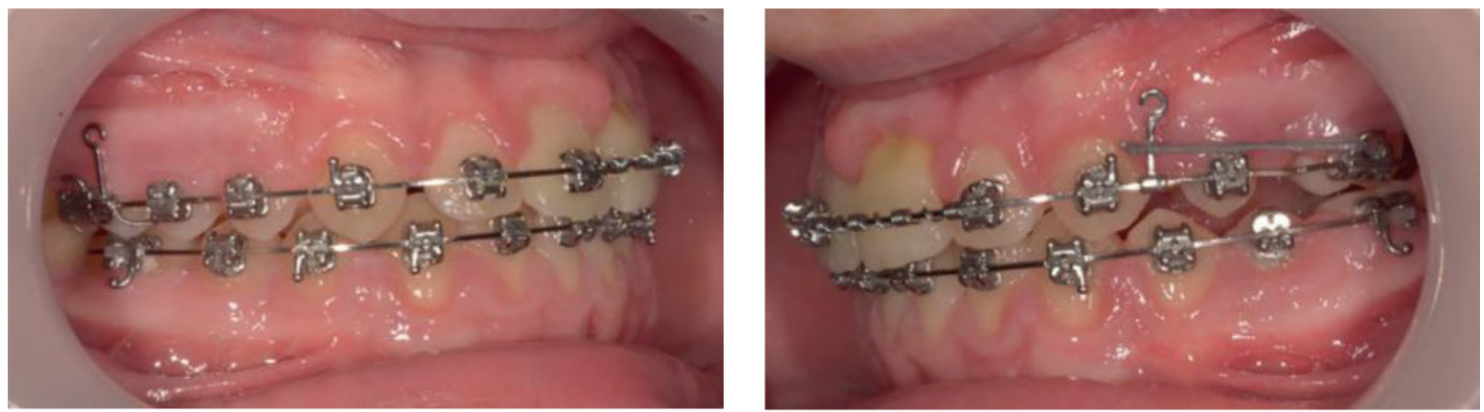

a

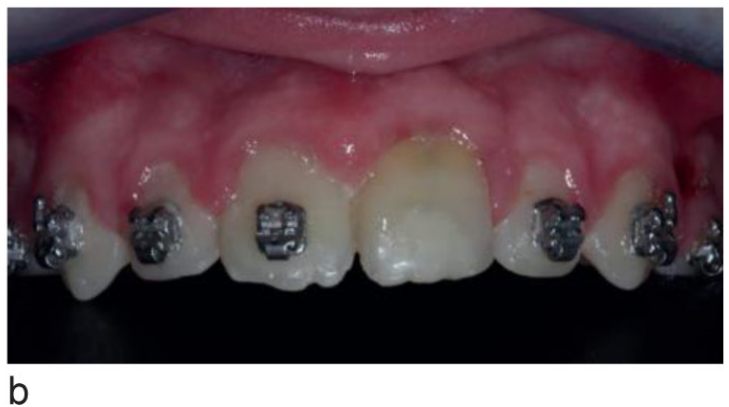

Figure 5

(a) Lateral view after reconstitution by stratification. The extraction space is being closed.

(b) Frontal view after reconstitution by stratification

The palatal cusp of the transplant was abraded in the same sitting to adapt its morphology to that of a central incisor (Fig. 5a, 5b).

To avoid any unwanted side effects, two miniscrews were placed in the extraction site. This area has the advantage of being isolated from any anatomical risk. These screws were used as anchors for the downward mesialization movement of the posterior teeth. At 6 months, a retroalveolar radiograph was obtained and there were no visible pathologies. Surface resorptions and replacements are usually visible as early as 4-8 weeks in transplantation cases. The inflammatory resorptions were visible at 2 months postoperatively. Clinically, the patient's tooth was asymptomatic and had physiological mobility. Periodontal

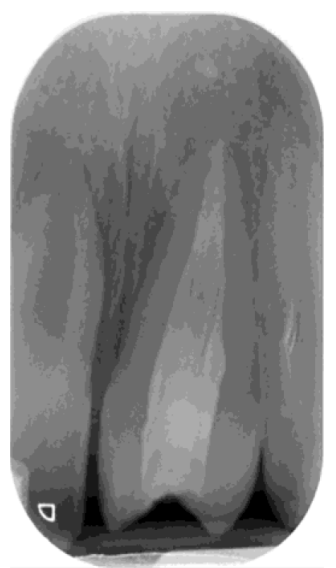

Figure 6

Radiographic follow-up at 6 months.

probing is negative. A bracket could then be bonded to the tooth to support it (Fig. 6). At the end of this treatment, the patient and his family were satisfied with the chosen treatment plan. 


\section{CONCLUSION}

Although not widely used, there are many advantages to using transplantation. However, only a seamless collaboration between the orthodontist, the dentist, and the oral surgeon will yield an optimal result. When making a decision on the treatment plan, the patient must be informed of the risk of failure inherent in any technique. Furthermore, it would be remiss of the practitioner, if the avulsion of teeth is indicated, not to propose autotransplantation, if necessary. It is important to note that teeth thus transplanted can be moved orthodontically. After treatment and the application of cosmetic camouflage, a long-term follow-up must be implemented.

Conflict of interest:The authors declare no conflicts of interest.

\section{BIBLIOGRAPHY}

1. Andreasen JO, Håkansson L. Atlas of replantation and transplantation of teeth. W.B. Saunders, 1992, $312 \mathrm{p}$.

2. Andreasen JO, Paulsen HU, Yu Z, Bayer T. A long-term study of 370 autotransplanted premolars. Part IV. Root development subsequent to transplantation. Eur J Orthod 1990;12(1):38-50.

3. Andreasen JO. Analysis of pathogenesis and topography of replacement root resorption (ankylosis) after replantation of mature permanent incisors in monkeys. Swed Dent J 1980;4(6):231-240.

4. Andreasen JO. Analysis of topography of surface and inflammatory root resorption after replantation of mature permanent incisors in monkeys. Swed Dent J 1980;4(4):135-144.

5. Andreasen JO. The effect of pulp extirpation or root canal treatment on periodontal healing after replantation of permanent incisors in monkeys. J Endod 1981;7(6):245-252.

6. Antonarakis GS, Prevezanos P, Gavric J, Christou P. Agenesis of maxillary lateral incisor and tooth replacement: cost-effectiveness of different treatment alternatives. Int J Prosthodont 2014;27(3):257-263.

7. Bauss O, Schilke R, Fenske C, Engelke W, Kiliaridis S. Autotransplantation of immature third molars: influence of different splinting methods and fixation periods. Dent Traumatol 2002;18(6):322-328.

8. Bauss O, Röhling J, Schwestka-Polly R. Prevalence of traumatic injuries to the permanent incisors in candidates for orthodontic treatment. Dent Traumatol 2004;20(2):61-66.

9. Cardesi E, Massei G, Cera G. Long-term histopathologic findings in tooth germs autotransplantation. Analysis of a series. Minerva Stomatol 1996;45(9):381-385.

10. Czochrowska EM, Stenvik A, Album B, Zachrisson BU. Autotransplantation of premolars to replace maxillary incisors: a comparison with natural incisors. Am J Orthod Dentofacial Orthop 2000;118(6):592-600.

11. Czochrowska EM, Stenvik A, Bjercke B, Zachrisson BU. Outcome of tooth transplantation: survival and success rates 17-41 years posttreatment. Am J Orthod Dentofacial Orthop 2002;121(2):110-119; quiz 193. 
12. Czochrowska EM, Stenvik A, Zachrisson BU. The esthetic outcome of autotransplanted premolars replacing maxillary incisors. Dent Traumatol 2002;18(5):237-245.

13. Garcia A. Apport des techniques d'autogreffes dentaires à I'orthodontie. Int Orthod 2005;3(3):221-234.

14. Gault PC, Warocquier-Clerout R. Tooth auto-transplantation with double periodontal ligament stimulation to replace periodontally compromised teeth. J Periodontol 2002;73(5):575-583.

15. Intra JBG, Roldi A, Brandão RCB, de Araújo Estrela CR, Estrela C. Autogenous premolar transplantation into artificial socket in maxillary lateral incisor site. J Endod 2014;1;40(11):1885-1890.

16. Kaku M, et al. A case of tooth autotransplantation after long-term cryopreservation using a programmed freezer with a magnetic field. Angle Orthod 2015;85(3):518-524.

17. Kim MS, Lee H-S, Nam OH, Choi SC. Auto-transplantation: A Reliable Treatment Modality for Severely Malpositioned Teeth. J Clin Pediatr Dent 2017;41(5):388-391.

18. Kim S, Lee S-J, Shin Y, Kim E. Vertical Bone Growth after Autotransplantation of Mature Third Molars: 2 Case Reports with Long-term Follow-up. J Endod 2015;41(8):1371-1374.

19. Osterne RL, Moreira Neto JJ, de Araújo Lima AD, Nogueira RL. Autotransplantation of Immature Third Molars and Orthodontic Treatment After En Bloc Resection of Conventional Ameloblastoma. J Oral Maxillofac Surg 2015;73(9):1686-1694.

20. Pogrel MA. Evaluation of over 400 autogenous tooth transplants. J Oral Maxillofac Surg 1987;45(3):205-211.

21. Reich PP. Autogenous transplantation of maxillary and mandibular molars. J Oral Maxillofac Surg 2008;66(11):2314-2317.

22. Temmerman L, De Pauw GA, Beele H, Dermaut LR. Tooth transplantation and cryopreservation: state of the art. Am J Orthod Dentofacial Orthop 2006;129(5):691-695.

23. Verweij JP, Anssari Moin D, Wismeijer D, van Merkesteyn JPR. Replacing Heavily Damaged Teeth by Third Molar Autotransplantation With the Use of Cone-Beam Computed Tomography and Rapid Prototyping. J Oral Maxillofac Surg 2017;75(9):1809-1816.

24. Yadav SS, Bodh R, Kaushik A, Talwar S. Auto-transplantation of a Buccally Erupted Matured Mandibular Third Molar to Replace a Grossly Decayed Second Molar. J Clin Diagn Res JCDR 2016;10(2):ZD06-07.

25. Yu HJ, Jia P, Lv Z, Qiu LX. Auto-transplantation of third molars with completely formed roots into surgically created sockets and fresh extraction sockets: a 10-year comparative study. Int J Oral Maxillofac Surg 2017;46(4):531-538.

26. Zufía J, Abella F, Trebol I, Gómez-Meda R. Auto-transplantation of Mandibular Third Molar with Buccal Cortical Plate to Replace Vertically Fractured Mandibular Second Molar: A Novel Technique. J Endod 2017;43(9):1574-1578. 